\title{
GENERALIZED WEYL'S THEOREM AND HYPONORMAL OPERATORS
}

\author{
M. BERKANI and A. ARROUD
}

(Received 20 July 2002; revised 10 March 2003)

Communicated by A. J. Pryde

\begin{abstract}
Let $T$ be a bounded linear operator acting on a Hilbert space $H$. The $B$-Weyl spectrum of $T$ is the set $\sigma_{B W}(T)$ of all $\lambda \in \mathbb{C}$ such that $T-\lambda I$ is not a $B$-Fredholm operator of index 0 . Let $E(T)$ be the set of all isolated eigenvalues of $T$. The aim of this paper is to show that if $T$ is a hyponormal operator, then $T$ satisfies generalized Weyl's theorem $\sigma_{B W}(T)=\sigma(T) \backslash E(T)$, and the $B$-Weyl spectrum $\sigma_{B W}(T)$ of $T$ satisfies the spectral mapping theorem. We also consider commuting finite rank perturbations of operators satisfying generalized Weyl's theorem.
\end{abstract}

2000 Mathematics subject classification: primary 47A53; secondary 47A55.

Keywords and phrases: $B$-Fredholm operator, $B$-Weyl spectrum, generalized Weyl's theorem.

\section{Introduction}

Let $X$ be a Banach space and let $L(X)$ be the Banach algebra of bounded linear operators acting on a Banach space $X$. For $T \in L(X)$, we denote by $N(T)$ the null space of $T$, by $\alpha(T)$ the nullity of $T$, by $R(T)$ the range of $T$ and by $\beta(T)$ its defect. If both $\alpha(T)$ and $\beta(T)$ are finite, then $T$ is called a Fredholm operator and the index of $T$ is defined by ind $(T)=\alpha(T)-\beta(T)$. In this case it is well known that the range $R(T)$ of $T$ is closed in $X$. Now for each nonnegative integer $n$ define $T_{n}$ to be the restriction of $T$ to $R\left(T^{n}\right)$ viewed as a map from $R\left(T^{n}\right)$ into $R\left(T^{n}\right)$ (in particular $T_{0}=T$ ). If for some $n$ the space $R\left(T^{n}\right)$ is closed and $T_{n}$ is a Fredholm operator, then $T$ is called a $B$-Fredholm operator [2, Definition 2.2]. In this case, by [2, Proposition 2.1], $T_{m}$ is a Fredholm operator and $\operatorname{ind}\left(T_{m}\right)=\operatorname{ind}\left(T_{n}\right)$ for each $m \geq n$. This remark leads to the following definition:

(C) 2004 Australian Mathematical Society 1446-7887/04 \$A2.00+0.00 
DEFINITION 1.1. Let $T \in L(X)$ be a $B$-Fredholm operator and let $n$ be any integer such that $T_{n}$ is a Fredholm operator. Then the index ind $(T)$ of $T$ is defined as the index of the Fredholm operator $T_{n}$.

In particular if $T$ is a Fredholm operator we find the usual definition of the index.

Let $B F(X)$ be the class of all $B$-Fredholm operators. In [2] the first author studied this class of operators and proved [2, Theorem 2.7] that an operator $T \in L(X)$ is a $B$-Fredholm operator if and only if $T=Q \oplus F$, where $Q$ is a nilpotent operator and $F$ is a Fredholm operator.

It is apparent from [6] that the concept of Drazin invertibility plays an important role for the class of $B$-Fredholm operators. If $A$ is an algebra with a unit 1 , following [16] we say that an element $x$ of $A$ is Drazin invertible if there is an element $b$ of $A$ and a nonnegative integer $k$ such that

$$
x^{k} b x=x^{k}, \quad b x b=b, \quad x b=b x .
$$

Recall that the concept of Drazin invertibility was originally introduced by Drazin in [9] where elements satisfying relation (1) are called pseudo-invertible elements. The Drazin spectrum of $a \in A$ is defined by

$$
\sigma_{D}(a)=\{\lambda \in \mathbb{C}: a-\lambda 1 \text { is not Drazin invertible }\} .
$$

In the case of a bounded linear operator $T$ acting on a Banach space $X$, it is well known that $T$ is Drazin invertible if and only if it has a finite ascent and descent (Definition 2.1); this is also equivalent to the fact that $T=U \oplus V$, where $U$ is an invertible operator and $V$ is nilpotent. (See [16, Proposition 6] and [12, Corollary 2.2].) In [6, Theorem 3.4] it is shown that $T$ is a $B$-Fredholm operator if and only if its projection in the algebra $L(X) / F_{0}(X)$ is Drazin invertible, where $F_{0}(X)$ is the ideal of finite rank operators in the algebra $L(X)$ of bounded linear operators acting on $X$. In [4], $B$-Weyl operators and $B$-Weyl spectrum were defined as follows:

Definition 1.2. Let $T \in L(X)$. Then $T$ is called a $B$-Weyl operator if it is a $B$-Fredholm operator of index 0 ; the $B$-Weyl spectrum $\sigma_{B W}(T)$ of $T$ is defined by $\sigma_{B W}(T)=\{\lambda \in \mathbb{C}: T-\lambda I$ is not a $B$-Weyl operator $\}$.

In the case of a normal operator $T$ acting on a Hilbert space $H$, the first author proved in [4, Theorem 4.5] that $\sigma_{B W}(T)=\sigma(T) \backslash E(T)$, where $E(T)$ is the set of all isolated eigenvalues of $T$, which gives a generalization of the classical Weyl's theorem. Recall that the classical Weyl's theorem [18] asserts that if $T$ is a normal operator acting on a Hilbert space $H$, then the Weyl spectrum $\sigma_{W}(T)$ is the set of all points in $\sigma(T)$ except the isolated eigenvalues of finite multiplicity, that is $\sigma_{W}(T)=\sigma(T) \backslash E_{0}(T)$, where $E_{0}(T)$ is the set of isolated eigenvalues of finite multiplicity, and $\sigma_{W}(T)$ is the Weyl 
spectrum of $T$, that is, the set of all $\lambda \in \mathbb{C}$ such that $\lambda I-T$ is not a Fredholm operator of index 0.

In his paper [1], Barnes considered version II of Weyl's theorem (called also Browder's theorem in [8]):

What conditions on $T$ imply that $T$ satisfies $\sigma_{W}(T)=\sigma(T) \backslash \Pi_{0}(T)$, where $\Pi_{0}(T)$ is the set of poles of the resolvent of $T$ of finite rank?

Recall that an isolated point $\lambda$ of the spectrum $\sigma(T)$ of $T$ is a pole of the resolvent of $T$ of finite rank if the spectral projection associated to the set $\{\lambda\}$ is of finite rank.

In [5] it is shown that if $T$ satisfies generalized Weyl's theorem $\sigma_{B W}(T)=$ $\sigma(T) \backslash E(T)$, then it satisfies Weyl's theorem $\sigma_{W}(T)=\sigma(T) \backslash E_{0}(T)$, and if it satisfies version II of generalized Weyl's theorem, $\sigma_{B W}(T)=\sigma(T) \backslash \Pi(T)$, then it satisfies version II of Weyl's theorem, $\sigma_{W}(T)=\sigma(T) \backslash \Pi_{0}(T)$, where $\Pi_{0}(T)$ is the set of the poles of the resolvent of $T$ of finite rank.

The aim of this paper is to consider generalized Weyl's theorem for hyponormal operators and to consider finite rank commuting perturbations for operators satisfying generalized Weyl's theorem. In the second section we show that if $T$ is a hyponormal operator acting on a Hilbert space $H$, then $T$ satisfies generalized Weyl's theorem $\sigma_{B W}(T)=\sigma(T) \backslash E(T)$ and the $B$-Weyl spectrum $\sigma_{B W}(T)$ of $T$ satisfies the spectral mapping theorem.

Moreover, if $f$ is an analytic function defined on a neighbourhood of the spectrum $\sigma(T)$ of $T$, then we show that $f(T)$ satisfies generalized Weyl's theorem, that is $\sigma_{B W}(f(T))=\sigma(f(T)) \backslash E(f(T))$. An analoguous result was obtained in the case of Weyl's spectrum by Oberai in [15], W. Y. Lee and S. H. Lee in [14], respectively in the case where $f$ is a polynomial or $f$ is an analytic function.

In the third section we consider an operator $T$ satisfying Weyl's theorem, a finite rank operator $F$, and we give a necessary and sufficient condition for $T+F$ to satisfy Weyl's theorem. This result gives an improvement of Oberai's result [15, Theorem 4].

Moreover, if $T$ satisfies generalized Weyl's theorem and $F$ commutes with $T$, we give a necessary and sufficient condition for $T+F$ to satisfy generalized Weyl's theorem. Furthermore we show that generalized Weyl's theorem holds for $T+F$ when $T$ is an isoloid or a quasinilpotent operator satisfying generalized Weyl's theorem and $F$ is a finite rank operator commuting with $T$.

\section{Generalized Weyl's theorem for hyponormal operators}

First we recall the following definition:

Definition 2.1. Let $T \in L(X), n \in \mathbb{N}$ and let

$$
c_{n}(T)=\operatorname{dim} R\left(T^{n}\right) / R\left(T^{n+1}\right), \quad c_{n}^{\prime}(T)=\operatorname{dim} N\left(T^{n+1}\right) / N\left(T^{n}\right) .
$$


Then the descent of $T$ is defined by

$$
\delta(T)=\inf \left\{n: c_{n}(T)=0\right\}=\inf \left\{n: R\left(T^{n}\right)=R\left(T^{n+1}\right)\right\},
$$

and the ascent of $T$ is defined by

$$
a(T)=\inf \left\{n: c_{n}^{\prime}(T)=0\right\}=\inf \left\{n: N\left(T^{n}\right)=N\left(T^{n+1}\right)\right\},
$$

with $\inf \emptyset=\infty$.

For $T \in L(X)$, let $\sigma_{B F}(T)=\{\lambda \in \mathbb{C}: T-\lambda I$ is not a $B$-Fredholm operator $\}$ be the $B$-Fredholm spectrum of $T$ and $\rho_{B F}(T)=\mathbb{C} \backslash \sigma_{B F}(T)$ the $B$-Fredholm resolvent set of $T$.

DEFINITION 2.2. Let $T \in L(X)$. We will say that $T$ is of stable sign index if for each $\lambda, \mu \in \rho_{B F}(T)$, ind $(T-\lambda I)$ and ind $(T-\mu I)$ have the same sign.

Recall that an operator $T \in L(H)$ on a Hilbert space $H$ is hyponormal if $T^{*} T-T T^{*} \geq 0$.

PROPOSITION 2.3. Let $H$ be a Hilbert space and let $T \in L(H)$ be a hyponormal operator. Then $T$ is of stable sign index.

Proof. Let $T$ be a hyponormal operator. Then for all $x \in H$ we have $\|T x\|^{2} \geq$ $\left\|T^{*} x\right\|^{2}$. So $N(T) \subset N\left(T^{*}\right)=R(T)^{\perp}$. Since $N\left(T^{2}\right) / N(T) \simeq N(T) \cap R(T)$, then $N\left(T^{2}\right)=N(T)$. Moreover, if $T$ is also a $B$-Fredholm operator, then there exists an integer $n$ such that $R\left(T^{n}\right)$ is closed and such that $T_{n}: R\left(T^{n}\right) \rightarrow R\left(T^{n}\right)$ is a Fredholm operator. We have

$$
\begin{aligned}
\operatorname{ind}(T)=\operatorname{ind}\left(T_{n}\right) & =\operatorname{dim} N(T) \cap R\left(T^{n}\right)-\operatorname{dim} R\left(T^{n}\right) / R\left(T^{n+1}\right) \\
& =-\operatorname{dim} R\left(T^{n}\right) / R\left(T^{n+1}\right) .
\end{aligned}
$$

So ind $(T) \leq 0$.

Further, if $\lambda \in \rho_{B F}(T)$, then $T-\lambda I$ is a $B$-Fredholm operator, and $T-\lambda I$ is also a hyponormal operator. From the preceding argument, we have ind $(T-\lambda I) \leq 0$. Therefore $T$ is of stable sign index.

THEOREM 2.4. Let $X$ be a Banach space, let $T \in L(X)$ be of stable sign index and let $f$ be a function analytic in a neighbourhood of the usual spectrum $\sigma(T)$ of $T$. Then $f\left(\sigma_{B W}(T)\right)=\sigma_{B W}(f(T))$. 
PROOF. If $\lambda \notin \sigma_{B W}(f(T))$, then $f(T)-\lambda I$ is a $B$-Fredholm operator of index 0 . So we can write $f(T)-\lambda I=\left(T-\mu_{1} I\right) \cdots\left(T-\mu_{r} I\right) g(T)$, where $\mu_{1}, \ldots, \mu_{r}$ are complex scalars and $g$ is an analytic function nonvanishing on the spectrum $\sigma(T)$ of $T$. In particular $g(T)$ is invertible. Since $f(T)-\lambda I$ is a $B$-Fredholm operator, from [2, Theorem 3.4] it follows that for each $i, 1 \leq i \leq r, T-\mu_{i} I$ is a $B$-Fredholm operator. Moreover, since ind $(f(T)-\lambda I)=0$ and $T$ is of stable sign index, then from [4, Theorem 3.2] we have for each $i, 1 \leq i \leq r, \operatorname{ind}\left(T-\mu_{i} I\right)=0$. So for each $i, 1 \leq i \leq r, \mu_{i} \notin \sigma_{B W}(T)$. If $\lambda \in f\left(\sigma_{B W}(T)\right)$, there exists $\mu \in \sigma_{B W}(T)$ such that $\lambda=f(\mu)$. Hence

$$
0=f(\mu)-\lambda=\left(\mu-\mu_{1}\right) \cdots\left(\mu-\mu_{r}\right) g(\mu)
$$

This implies that $\mu \in\left\{\mu_{1}, \ldots, \mu_{r}\right\}$. So there exists $i, 1 \leq i \leq r$, such that $\mu_{i} \in$ $\sigma_{B W}(T)$, and this is a contradiction. Hence $\lambda \notin f\left(\sigma_{B W}(T)\right)$.

Conversely suppose that $\lambda \notin f\left(\sigma_{B} w(T)\right)$. If $\lambda \in \sigma_{B W}(f(T))$ then $\lambda \in \sigma(f(T))=$ $f(\sigma(T))$. Hence there exists $\mu \in \sigma(T)$ such that $\lambda=f(\mu)$. We have

$$
f(T)-\lambda I=f(T)-f(\mu) I=\left(T-\mu_{1} I\right) \cdots\left(T-\mu_{r} I\right) g(T),
$$

where $\mu_{1}, \ldots, \mu_{r}$ are complex scalars and $g$ is an analytic function nonvanishing on the spectrum $\sigma(T)$ of $T$. Since $f(T)-\lambda I$ is not a $B$-Fredholm operator of index 0 , from [2, Theorem 3.4] and [4, Theorem 3.2] there exists $\alpha \in\left\{\mu_{1}, \ldots, \mu_{r}\right\}$ such that $T-\alpha I$ is not a $B$-Fredholm operator of index 0 . So $\lambda=f(\alpha)$ and $\lambda \in f\left(\sigma_{B W}(T)\right)$. This contradicts our assumption. Consequently, $\lambda \notin \sigma_{B W}(f(T))$ and $f\left(\sigma_{B W}(T)\right)=\sigma_{B W}(f(T))$.

Since a hyponormal operator is of stable sign index, we have immediately the following corollary.

COROLLARY 2.5. Let $H$ be a Hilbert space, let $T \in L(H)$ be a hyponormal operator and let $f$ be a function analytic in a neighbourhood of the usual spectrum $\sigma(T)$ of $T$. Then $f\left(\sigma_{B W}(T)\right)=\sigma_{B W}(f(T))$.

It is proved in [4, Theorem 4.5] that a normal operator acting on a Hilbert space $H$ satisfies generalized Weyl's theorem $\sigma_{B W}(T)=\sigma(T) \backslash E(T)$. In the following theorem, we extend this result to the case of a hyponormal operator.

THEOREM 2.6. Let $H$ be a Hilbert space and let $T \in L(H)$ be a hyponormal operator. Then $T$ satisfies generalized Weyl's theorem $\sigma_{B W}(T)=\sigma(T) \backslash E(T)$.

PROOF. If $\lambda \in \sigma(T)$ and $\lambda \notin \sigma_{B W}(T)$, then $T-\lambda I$ is a $B$-Fredholm operator of index 0. From [4, Lemma 4.1], there exist two closed subspaces $M, N$ of $H$ such that 
$H=M \oplus N$, and $T-\lambda I=U \oplus V$ with $U=(T-\lambda I)_{\mid M}$ a Fredholm operator of index 0 and $V=(T-\lambda I)_{\mid N}$ a nilpotent operator.

Let $S=T_{\mid M}$ and $I_{M}=I_{\mid M}$. Since $T$ is a hyponormal operator, then $S$ is also a hyponormal operator and $S-\lambda I_{\mid M}=U$ is a Fredholm operator of index 0 .

If $\lambda \in \sigma(S)$, since $S$ is a hyponormal operator, from [7, Theorem 3.1] we have $\sigma_{W}(S)=\sigma(S) \backslash E_{0}(S)$. As $\lambda \notin \sigma_{W}(S)$ we have $\lambda \in E_{0}(S)$. In particular, $\lambda$ is isolated in $\sigma(S)$. Since $T-\lambda I=U \oplus V=\left(S-\lambda I_{\mid M}\right) \oplus V$, and $V$ is a nilpotent operator, we have $\sigma(U) \backslash\{0\}=\sigma(T-\lambda I) \backslash\{0\}$. Therefore 0 is isolated in $\sigma(T-\lambda I)$ or equivalently $\lambda$ is isolated in $\sigma(T)$. As $\lambda \in E_{0}(S)$ then $\lambda \in E(T)$.

If $\lambda \notin \sigma(S)$, then $T-\lambda I$ is Drazin invertible, and $\lambda$ is isolated in $\sigma(T)$. Since $T-\lambda I$ is not invertible, we have $\lambda \in E(T)$.

Conversely, if $\lambda \in E(T)$, then $\lambda$ is isolated in $\sigma(T)$. From [11, Theorem 7.1] we have $X=M \oplus N$, where $M, N$ are closed subspaces of $X, U=(T-\lambda I)_{\mid M}$ is an invertible operator and $V=(T-\lambda I)_{\mid N}$ is a quasinilpotent operator. Since $T$ is a hyponormal operator, then $V$ is also a hyponormal operator. As $V$ is quasinilpotent, from [17, Chapter XI, Theorem 5.1] we have $V=0$. Therefore $T-\lambda I$ is Drazin invertible. By [4, Lemma 4.1] $T-\lambda I$ is a $B$-Fredholm operator of index 0 .

Now we consider a Hilbert space $H$, an operator $T \in L(H)$, and a function $f$ analytic in a neighbourhood of the spectrum $\sigma(T)$ of $T$. In [14] it has been proved that if $T$ is a hyponormal operator, then Weyl's theorem holds for $f(T)$. We prove now that generalized Weyl's theorem holds also for $f(T)$. We begin with the following lemma.

LEMMA 2.7. Let $X$ be a Banach space and let $T \in L(X)$. Then

$$
\sigma(f(T)) \backslash E(f(T)) \subset f[\sigma(T) \backslash E(T)] .
$$

PROOF. If $\lambda \in \sigma(f(T)) \backslash E(f(T))$ then $\lambda \in \sigma(f(T))=f(\sigma(T))$.

(a) If $\lambda$ is not isolated in $f(\sigma(T))$, then there exists an infinite sequence $\left(\mu_{n}\right)_{n \in \mathbb{N}} \subset$ $\sigma(T)$ such that $f\left(\mu_{n}\right) \rightarrow \lambda$. Since $\sigma(T)$ is compact, we may assume that $\left(\mu_{n}\right)_{n \in \mathbb{N}}$ converges to $\mu_{0}$ in $\sigma(T)$. It follows that $\mu_{0}$ is not isolated in $\sigma(T)$ and $\lambda=f\left(\mu_{0}\right)$. Hence $\lambda \in f[\sigma(T) \backslash E(T)]$.

(b) Now suppose that $\lambda$ is isolated in $f(\sigma(T))$. Since $\lambda \notin E(f(T))$, then $\lambda$ is not an eigenvalue of $f(T)$. We can write

$$
f(T)-\lambda I=\left(T-\mu_{1} I\right) \cdots\left(T-\mu_{r} I\right) g(T),
$$

where $\mu_{1}, \ldots, \mu_{r}$ are complex scalars and $g(T)$ is an invertible operator. As $\lambda \notin$ $E(f(T))$, then for all $\mu \in\left\{\mu_{1}, \ldots, \mu_{r}\right\}, \mu$ is not an eigenvalue of $T$. Since $f(T)-\lambda I$ is not invertible, there exists $\mu \in\left\{\mu_{1}, \ldots, \mu_{r}\right\}$ such that $T-\mu I$ is not invertible. Hence $f(\mu)=\lambda$ and $\lambda \in f[\sigma(T) \backslash E(T)]$. 
DEFINITION 2.8 (See [15]). Let $X$ be a Banach space. An operator $T \in L(X)$ is said to be an isoloid if iso $\sigma(T) \subseteq E(T)$, where iso $\sigma(T)$ is the set of isolated points in $\sigma(T)$.

LEMMA 2.9. Let $X$ be a Banach space and let $T \in L(X)$. If $T$ is an isoloid, then $\sigma(f(T)) \backslash E(f(T))=f[\sigma(T) \backslash E(T)]$.

PROOF. Let us prove that $f[\sigma(T) \backslash E(T)] \subset \sigma(f(T)) \backslash E(f(T))$. If $\lambda \in \sigma(f(T)) \cap$ $E(f(T))$, then $f(T)-\lambda I=\left(T-\mu_{1} I\right)^{m_{1}} \cdots\left(T-\mu_{r} I\right)^{m_{r}} g(T)$, where $m_{1}, \ldots, m_{r}$ are integers, $\mu_{1}, \ldots, \mu_{r}$ are complex scalars, $g(T)$ is an invertible operator, and $\mu_{i} \neq \mu_{j}$ for $i \neq j$. Since $f(T)-\lambda I$ is not invertible, there exists $\mu \in\left\{\mu_{1}, \ldots, \mu_{r}\right\}$ such that $\mu \in \sigma(T)$. Since $\lambda$ is isolated in $\sigma(f(T)), \mu$ is isolated in $\sigma(T)$. Hence $\lambda=f(\mu) \notin f[\sigma(T) \backslash E(T)]$. Therefore $f[\sigma(T) \backslash E(T)] \subset \sigma(f(T)) \backslash E(f(T))$. From Lemma 2.7 we know that $\sigma(f(T)) \backslash E(f(T)) \subset f[\sigma(T) \backslash E(T)]$. Hence

$$
\sigma(f(T)) \backslash E(f(T))=f[\sigma(T) \backslash E(T)] .
$$

THEOREM 2.10. Let $X$ be a Banach space, let $T \in L(X)$ be an isoloid operator which satisfies generalized Weyl's theorem, let f be a function analytic in a neighbourhood of the spectrum $\sigma(T)$ of $T$. Then generalized Weyl's theorem holds for $f(T)$ if and only if $f\left(\sigma_{B W}(T)\right)=\sigma_{B W}(f(T))$.

Proof. Since $T$ is an isoloid, $\sigma(f(T)) \backslash E(f(T))=f[\sigma(T) \backslash E(T)]$. Moreover, as generalized Weyl's theorem holds for $T, \sigma_{B W}(T)=\sigma(T) \backslash E(T)$. Hence $f\left(\sigma_{B W}(T)\right)=f[\sigma(T) \backslash E(T)]=\sigma(f(T)) \backslash E(f(T))$. So generalized Weyl's theorem holds for $f(T)$ if and only if $f\left(\sigma_{B W}(T)\right)=\sigma_{B W}(f(T))$.

COROLlary 2.11. Let $H$ be a Hilbert space, let $T \in L(H)$ be a hyponormal operator and let fbe a function analytic in a neighbourhood of the spectrum $\sigma(T)$ of $T$. Then $f(T)$ satisfies generalized Weyl's theorem $\sigma_{B W}(f(T))=\sigma(f(T)) \backslash E(f(T))$.

PROOF. A hyponormal operator on a Hilbert space satisfies generalized Weyl's theorem and it is well known that a hyponormal operator is an isoloid. Moreover, from Theorem 2.4 we have $\sigma_{B W}(f(T))=f\left(\sigma_{B W}(T)\right)$. From Theorem 2.10, it follows that $f(T)$ satisfies generalized Weyl's theorem.

\section{Finite rank perturbation and generalized Weyl's theorem}

In this part we consider an operator $T$ satisfying generalized Weyl's theorem and a finite rank operator $F$ commuting with $T$, and we give a necessary and sufficient condition for $T+F$ to satisfy generalized Weyl's theorem. Moreover, we obtain 
similar results as those obtained in the case of Weyl's theorem in [10,13] and [15]. We begin with the case of Weyl's theorem and we give an improvement of Oberai's Theorem [15, Theorem 4].

THEOREM 3.1. Let $X$ be a Banach space and let $T \in L(X)$. If $T$ satisfies Weyl's theorem and $F$ is a finite rank operator in $L(X)$, then $T+F$ satisfies Weyl's theorem if and only if $\Pi_{0}(T+F)=E_{0}(T+F)$.

PROOF. If $T+F$ satisfies Weyl's theorem, then from [1, Corollary 5], we have $\Pi_{0}(T+F)=E_{0}(T+F)$. Conversely if $\Pi_{0}(T+F)=E_{0}(T+F)$, since $T$ satisfies Weyl's theorem, then from [1, Corollary 5] we have $E_{0}(T)=\Pi_{0}(T)$. Since $F$ is a finite rank operator, from [4, Theorem 4.3] we have $\sigma_{W}(T+F)=\sigma_{W}(T)$. If $F$ commutes with $T$, we have also $\sigma_{B}(T+F)=\sigma_{B}(T)$, where $\sigma_{B}(T)$ is the Browder spectrum of $T$ (see [1]). Since $T$ satisfies Weyl's theorem, then $\sigma_{W}(T+F)=$ $\sigma_{W}(T)=\sigma_{B}(T)=\sigma_{B}(T+F)$. As we have $\Pi_{0}(T+F)=E_{0}(T+F)$, then from [1, Corollary 5], $T+F$ satisfies Weyl's theorem. If $F$ does not commute with $T$, then we use the same argument as Oberai in [15, Theorem 4].

THEOREM 3.2. Let $X$ be a Banach space and let $T \in L(X)$. If $T$ satisfies generalized Weyl's theorem and $F$ is a finite rank operator in $L(X)$ commuting with $T$, then $T+F$ satisfies generalized Weyl's theorem if and only if $\Pi(T+F)=E(T+F)$.

Proof. If $T+F$ satisfies generalized Weyl's theorem, then from [3, Corollary 2.6], we have $\Pi(T+F)=E(T+F)$. Conversely if $\Pi(T+F)=E(T+F)$, since $T$ satisfies generalized Weyl's theorem, then $\sigma_{B W}(T)=\sigma_{D}(T)$. Since $F$ is a finite rank operator, from [4, Theorem 4.3] we have $\sigma_{B W}(T)=\sigma_{B W}(T+F)$. As $F$ commutes with $T$, from [3, Theorem 2.7] we have $\sigma_{D}(T)=\sigma_{D}(T+F)$. So $\sigma_{B W}(T+F)=$ $\sigma_{D}(T+F)$. Since $\Pi(T+F)=E(T+F)$, then from [3, Corollary 2.6] $T+F$ satisfies generalized Weyl's theorem.

The following lemma is useful in the proof of the next two theorems.

LEMMA 3.3 ([13, Lemma 2.1]). Let $T \in L(X)$. If $F \in L(X)$ is a finite rank operator, then $\operatorname{dim} N(T)<\infty \Longleftrightarrow \operatorname{dim} N(T+F)<\infty$. Moreover, if $F$ commutes with $T$, then $\lambda \in \operatorname{acc} \sigma(T) \Longleftrightarrow \lambda \in \operatorname{acc} \sigma(T+F)$, where $\operatorname{acc} \sigma(T)$ is the set of the accumulation points of $\sigma(T)$.

THEOREM 3.4. Let $T \in L(X)$ be an isoloid operator and $F \in L(X)$ be a finite rank operator commuting with $T$. If $T$ satisfies generalized Weyl's theorem, then $T+F$ satisfies generalized Weyl's theorem. 
ProOF. In view of Theorem 3.2 it is enough to show that $\Pi(T+F)=E(T+F)$. Since $\Pi(T+F) \subset E(T+F)$ is always true, we only have to prove that $\Pi(T+F) \supset$ $E(T+F)$.

If $\lambda \in E(T+F)$, then $\lambda$ is isolated in $\sigma(T+F)$ and according to Lemma 3.3, $\lambda$ is isolated in $\sigma(T)$. Since $T$ satisfies generalized Weyl's theorem, it follows that $\lambda \in E(T)=\Pi(T)$. Finally since $\Pi(T)=\Pi(T+F)$ we have $\lambda \in \Pi(T+F)$.

REMARK 3.5. Let $T \in L(X)$. If $T$ has no eigenvalues, then $T$ satisfies generalized Weyl's theorem. To prove this, assume that $\lambda \in \sigma(T)$; for simplicity assume $\lambda=0$. If $0 \notin \sigma_{B W}(T)$, then $T$ is a $B$-Fredholm operator of index 0 . Hence there is an integer $n$, such that $R\left(T^{n}\right)$ is closed and ind $(T)=\operatorname{ind}\left(T_{n}\right)=\operatorname{dim} N(T) \cap R\left(T^{n}\right)-$ $\operatorname{dim} R\left(T^{n}\right) / R\left(T^{n+1}\right)=0$. Since $N(T)=0$, then $R\left(T^{n}\right)=R\left(T^{n+1}\right)$ and then $X=R(T)$. So $T$ is invertible, and this is a contradiction with our hypothesis. Hence $\sigma_{B W}(T)=\sigma(T)$ and $T$ satisfies generalized Weyl's theorem.

PROPOSITION 3.6. Let $X$ be a Banach space and let $T \in L(X)$. If $T$ satisfies generalized Weyl's theorem and $N$ is a finite rank nilpotent operator in $L(X)$ commuting with $T$, then $T+N$ satisfies generalized Weyl's theorem.

ProOF. Let us prove that if $\lambda$ is an eigenvalue of $T$ then $\lambda$ is also an eigenvalue of $T+N$. We may assume that $\lambda=0$. Then there exists $x \neq 0$ and $m \in \mathbb{N}$ such that $T x=0$ and $N^{m}=0$. We have

$$
(T+N)^{m} x=\sum_{k=0}^{m}\left(\begin{array}{l}
m \\
k
\end{array}\right) T^{k} N^{m-k} x=0 .
$$

So there exists $p \in \mathbb{N}, p \leq m$, such that $(T+N)^{p} x \neq 0$ and that $(T+N)(T+$ $N)^{p} x=0$. Hence 0 is an eigenvalue of $T+N$ and $E(T) \subset E(T+N)$. By symmetry we have $E(T)=E(T+N)$. If $\lambda \notin \sigma_{B W}(T)$, then $T-\lambda I$ is $B$-Fredholm of index 0 . From [4, Proposition 3.3], since $N$ is of finite rank, it follows that $T+N-\lambda I$ is also a $B$-Fredholm operator of index 0 . So $\lambda \notin \sigma_{B W}(T+N)$. By symmetry we have $\sigma_{B W}(T+N)=\sigma_{B W}(T)$. Since $\sigma(T+N)=\sigma(T)$, then $T+N$ satisfies generalized Weyl's theorem.

EXAMPLE 1 ([15, Example 2]). Let $H=\ell_{2}$ and let $T$ and $N$ in $L(H)$ be defined by

$$
\begin{aligned}
T\left(x_{1}, x_{2}, x_{3}, \ldots\right) & =\left(0, x_{1} / 2, x_{2} / 3, \ldots\right) \\
N\left(x_{1}, x_{2}, x_{3}, \ldots\right) & =\left(0,-x_{1} / 2,0,0, \ldots\right)
\end{aligned}
$$

Since $T$ has no eigenvalues, from the Remark 3.5 the operator $T$ satisfies generalized Weyl's theorem. So from [5, Theorem 3.9] $T$ satisfies also Weyl's theorem. Also $N$ 
is a nilpotent operator of finite rank. But from [15, Example 2], the operator $T+N$ does not satisfy Weyl's theorem and so from [5, Theorem 3.9] it does not satisfy generalized Weyl's theorem either. This example shows that Proposition 3.6 may not hold if $N$ does not commute with $T$.

REMARK 3.7. Let $T \in L(X)$ be a quasinilpotent operator and $F \in L(X)$ be a finite rank operator commuting with $T$. If $T$ is injective then $F$ is nilpotent. To see this, and under these conditions, $T F$ is a finite rank quasinilpotent operator, therefore $T F$ is a nilpotent operator. As $T$ is injective, then $F$ is also a nilpotent operator.

THEOREM 3.8. Let $T \in L(X)$ be a quasinilpotent operator and $F \in L(X)$ a finite rank operator commuting with $T$. If $T$ satisfies generalized Weyl's theorem, then $T+F$ satisfies generalized Weyl's theorem.

Proof. If $T$ is injective, then by Remark 3.7, $F$ is a nilpotent operator and the result follows from Proposition 3.6.

Suppose $T$ is not injective. Since $T$ satisfies generalized Weyl's theorem, then by [5, Theorem 3.9 ], $T$ also satisfies Weyl's theorem. Hence $\sigma_{W}(T)=\sigma(T) \backslash E_{0}(T)$. As $T$ is a quasinilpotent operator, then $\sigma_{W}(T)=\{0\}$. It follows that $E_{0}(T)=\emptyset$ and, since $T$ is not injective, $\operatorname{dim} N(T)=\infty$. This implies by Lemma 3.3 that $\operatorname{dim} N(T+F)=\infty$. It is easily seen that $\sigma(T+F)=\sigma(F)=\left\{0, \lambda_{1}, \ldots, \lambda_{k}\right\}$, where $\lambda_{i}, i=1, \ldots, k$, are the non-zero scalars of the spectrum of $F$ when they exist. We have also $E(T+F)=\left\{0, \lambda_{1}, \ldots, \lambda_{k}\right\}$.

Since

$$
\sigma_{B W}(T)=\sigma_{B W}(T+F) \quad \text { and } \quad \sigma_{B W}(T)=\sigma(T) \backslash E(T)=\emptyset,
$$

we have $\sigma_{B w}(T+F)=\sigma(T+F) \backslash E(T+F)$.

LemMA 3.9. Let $T \in L(X)$, let $X=M \oplus N$ where $M, N$ are two closed subspaces of $X$, and let $U=T_{\mid M}, V=T_{\mid N}$. If $T$ is a B-Fredholm operator, then $U, V$ are $B$-Fredholm operators.

Proof. Let us prove that $V$ is a $B$-Fredholm operator. Let $P$ be the projection of $X$ onto $N$ along $M$. Clearly $P$ is a $B$-Fredholm operator and commutes with $T$. Then by [6, Corollary 3.5], $T P$ is a $B$-Fredholm operator. Consequently there is an integer $n$ such that $R\left((T P)^{n}\right)$ is closed and $(T P)_{n}: R\left((T P)^{n}\right) \rightarrow R\left((T P)^{n}\right)$ is Fredholm.

Since $R\left((T P)^{n}\right)=R\left(V^{n}\right)$ and $(T P)_{n}=V_{n}$, then $V$ is a $B$-Fredholm operator.

EXAMPLE 2. Let $S$ be an injective quasinilpotent operator which is not nilpotent on the Hilbert space $\ell_{2}$. We define $T$ on $\ell_{2} \oplus \ell_{2}$ by $T=I \oplus S$ where $I$ is the identity on $\ell_{2}$. It follows easily that $\sigma(T)=\{0,1\}$ and $E(T)=\{1\}$. Let us prove that $\sigma_{B W}(T)=\{0\}$. 
We have $T-(I \oplus I)=0 \oplus(S-I)$ and since $S-I$ is an invertible operator, $T-(I \oplus I)$ is a $B$-Fredholm operator of index 0 , and $1 \notin \sigma_{B W}(T)$.

Suppose that $T$ is a $B$-Fredholm operator. Then by Lemma 3.9, $S$ is a $B$-Fredholm operator. From [2, Theorem 2.7], there exist two closed $S$-invariant subspaces of $\ell_{2}$, $M$ and $N$ such that $\ell_{2}=M \oplus N$ and $S=U \oplus V$ where $U=S_{\mid M}$ is nilpotent and $V=S_{\mid N}$ is invertible.

If $m$ is a sufficiently large integer, we have $U^{m}=0$ and $S^{m}=U^{m} \oplus V^{m}=0 \oplus V^{m}$. Hence $\sigma\left(V^{m}\right) \subset \sigma\left(S^{m}\right)=\{0\}$. But since $V$ is invertible, we have $N=0$ and then $S=U$ is nilpotent, which contradicts the hypothesis on $S$. So $\sigma_{B W}(T)=\{0\}$ and $\sigma_{B W}(T)=\sigma(T) \backslash E(T)$. Hence $T$ satisfies generalized Weyl's theorem.

We define the operator $K$ on $\ell_{2}$ by $K\left(x_{1}, x_{2}, \ldots\right)=\left(-x_{1}, 0,0, \ldots\right)$ and $F=K \oplus 0$ on $\ell_{2} \oplus \ell_{2}$. Then $F$ is a finite rank operator and we have $\sigma(T+F)=\{0,1\}$ and $E(T+F)=\{0,1\}$.

As $\sigma_{B W}(T+F)=\sigma_{B W}(T)=\{0\}$, then $T+F$ does not satisfy generalized Weyl's theorem.

\section{Acknowledgment}

The authors would like to thank the referee for several helpful remarks and comments.

\section{References}

[1] B. A. Barnes, 'Riesz points and Weyl's theorem', Integral Equations Operator Theory 34 (1999), 187-196.

[2] M. Berkani, 'On a class of quasi-Fredholm operators', Integral Equations Operator Theory 34 (1999), 244-249.

[3] — ' $B$-Weyl spectrum and poles of the resolvent', J. Math. Anal. Appl. 272 (2002), 596-603.

[4] _ 'Index of $B$-Fredholm operators and generalization of a Weyl theorem', Proc. Amer. Math. Soc. 130 (2002), 1717-1723.

[5] M. Berkani and J. J. Koliha, 'Weyl type theorems for bounded linear operators', Acta Sci. Math. (Szeged) 69 (2003), 359-376.

[6] M. Berkani and M. Sarih, 'An Atkinson's type theorem for $B$-Fredholm operators', Studia Math. 148 (2001), 251-257.

[7] L. A. Coburn, 'Weyl's theorem for nonnormal operators', Michigan Math. J. 13 (1966), 285-288.

[8] S. V. Djordjević and Y. M. Han, 'Browder's theorems and spectral continuity', Glasgow Math. J. $42(2000), 479-486$.

[9] M. P. Drazin, 'Pseudoinverse in associative rings and semigroups', Amer. Math. Monthly 65 (1958), 506-514.

[10] J.-C. Hou and X.-L. Zhang, 'On the Weyl spectrum: Spectral mapping theorem and Weyl's theorem', J. Math. Anal. Appl. 220 (1998), 760-768.

[11] J. J. Koliha, 'A generalized Drazin inverse', Glasgow Math. J. 38 (1996), 367-381. 
[12] D. C. Lay, 'Spectral analysis using ascent, descent, nullity and defect', Math. Ann. 184 (1970), 197-214.

[13] S. H. Lee and W. Y. Lee, 'On Weyl's theorem II', Math. Japon. 43 (1996), 549-553.

[14] W. Y. Lee and S. H. Lee, 'A spectral mapping theorem for the Weyl spectrum', Glasgow Math. J. 38 (1996), 61-64.

[15] K. K. Oberai, 'On the Weyl spectrum II', Illinois J. Math. 21 (1977), 84-90.

[16] S. Roch and B. Silbermann, 'Continuity of generalized inverses in Banach algebras', Studia Math. 136 (1999), 197-227.

[17] M. Schechter, Principles of functional analysis (Academy Press, New York, 1971).

[18] H. Weyl, 'Über beschränkte quadratische Formen, deren Differenz vollstetig ist', Rend. Circ. Mat. Palermo 27 (1909), 373-392.

Groupe d'Analyse et Théorie des Opérateurs (G.A.T.O.)

Université Mohammed I

Faculté des Sciences

Département de Mathématiques

Oujda

Morocco

e-mail: berkani@sciences.univ-oujda.ac.ma arroud@sciences.univ-oujda.ac.ma 